\title{
eICIC Optimization Improvements in Downlink Resource Allocation in LTE-A HetNets
}

\author{
Júnio Moreira (D), Éderson R. Silva (D), and Paulo R. Guardieiro, Member, IEEE
}

\begin{abstract}
Resource allocation mechanisms and management of inter-cell interference coordination (ICIC) are fundamental characteristics of the LTE-A network for achieving maximum capacity. Cell densification serves as a promising candidate solution for meeting the demand posed by mobile users, as well as optimizing coverage. However, the transmit power difference between the introduced picocells and the legacy macrocells essentially leads to challenges that limits system performance, especially for those users located at the edge of the cell. As a means of providing a clear understanding, the authors thus formulate the optimization problem as game theory, in order to maximize a modified utility function, which aims at making improvements on the eICIC. Based on exact potential game formulation, we optimize almost blank subframe (ABS) and cell selection bias (CSB) settings for both macrocells and picocells in a distributed manner. Simulation results illustrate important performance gains on the service fairness of the users, and especially for cell edge users, where the averaged throughput increases by up to $51 \%$, when compared to eICIC optimization without our modified utility function.
\end{abstract}

Index Terms-Resource Allocation, Inter-Cell Interference, LTE-A Networks, HetNets, Game Theory.

\section{INTRODUCTION}

W ITH the rise of increasingly agile and efficient media [1], the high quality of service (QoS) has attracted the attention of mobile operators and the research areas in recent years. In this context, the Third Generation Partnership Project (3GPP) introduced the Long Term Evolution-Advanced (LTEA) standard [2]. In the LTE-A network, the main objective is to achieve higher network capacity compared to that of LTE network.

Next generation cellular systems promise a significant increase in cell throughput and improved spectral efficiency compared to current systems. To achieve these goals, network densification is a key element in increasing system coverage and meeting the growing demands for mobile data in a costeffective manner due to its capability of frequency spectrum reuse and signal-to-interference-plus-noise ratio (SINR) [3].

As a result, inter-cell interference (ICI) issues arise that have a negative impact on user equipament (UE) throughput and system performance. ICI coordination (ICIC) techniques are proposed for mitigating ICI and improving UEs throughput without reducing spectral efficiency.

Júnio Moreira is with the Federal Institute of Triângulo Mineiro (e-mail: juniomoreira@iftm.edu.br). Éderson R. Silva and Paulo R. Guardieiro are with the Federal University of Uberlândia (e-mail: \{ersilva, prguardieiro\} @ufu.br).

The authors would like to thank FAPEMIG (Minas Gerais Research Foundation, Brazil), this work was supported in part by under Grant TECAPQ-03279-17.

Digital Object Identifier (DOI): 10.14209/jcis.2020.2
In a heterogeneous networks (HetNets) architecture, the coverage area of a macrocell is overlaid with several lowpower access nodes, such as picocells, femtocells, and relays [4]. The transmission power of macrocells is much higher than that of picocells, so mobile users who are physically located near picocells may be attracted to macrocells, which can create underutilized picocells and overcrowded macrocells. On the other hand, the surrounding macrocells of a picocell can generate higher rates of interference for a user associated with the picocell. Therefore, in these networks, smart user association, resource allocation, and interference management schemes are required for achieving performance gains. However, to tackle these issues, enhanced inter-cell interference coordination (eICIC) has been introduced in 3GPP Release10 [5]. Two representative techniques are briefly summarized below.

(i) Almost blank subframe (ABS) is configured on macrocells so that the macrocell ceases data transmissions in certain time slots, which reduces interference on picocells, and

(ii) Cell selection bias (CSB) is used to balance the received signal power from macrocells to a user so that a user is not necessarily associated with the macrocell that provides the strongest received power.

The use of ABS patterns can help reduce the interference from macrocells to picocells. To achieve the maximum possible performance gain using eICIC, joint optimization in ABS patterns and CSB values is required [6]. However, by ceasing transmission at certain timeslots using ABS, the eICIC can reduce the proper utilization of macrocell resources.

In light of the aforementioned, in this paper, the authors provide a distributed algorithm that jointly optimizes the ABS patterns and CSB values in HetNets. We formulate the problem as an exact potential game, through the adaptation of the game theoretic frameworks found in [7] as well as propose some improvements to these algorithms in order to achieve better performance, called eICIC+. Through simulation studies, we show that the proposed optimization can deliver improvements of around $51 \%$ over average user throughput. In turn, service fairness among users can also be greatly improved.

In summary, the main contributions of this paper are the following.

- We propose an extension of the eICIC optimization that uses an exact potential game, through use of a modified utility function (including statistics based on past throughput history and weight input ( $\beta$ and $\gamma)$ ).

- The authors also investigate and compare the performance of different schedulers for downlink channel in LTE- 
A HetNets. We evaluate the performance with Matlab simulations in order to demonstrate the relevance of the approach, mainly for cell edge users.

- Finally, the robustness of the eICIC+ optimization is tested against the eICIC scheme. We provide an analytical investigation of the algorithm eICIC+ and comprehensive performance study regarding eICIC.

This paper is organized as follows. Section II reviews literature on eICIC optimization as well as studies that apply game theory to this problem. We then introduce an overview on eICIC on LTE-A networks in Section III, while system modeling is presented in Section IV. The proposed algorithm, as well as the downlink schedulers used in this paper are described in Section V and the evaluation results are presented and discussed in Section VI, followed by the evaluation scenario. Finally, in Section VII we draw our conclusions and highlight some potential future studies.

\section{RELATED WORK}

This section reviews literature related to eICIC optimization in LTE-A HetNets, highlighting the contributions reached from our proposed solution.

Several algorithms for elCIC optimization, ABS patterns and CSB values are proposed in the literature. Disjoint optimization of ABS and CSB is performed in [8], where the ABS patterns are simplified as fractional numbers, and the convergence of the algorithms is proven using stochastics theorems. The authors of the paper presented in [9] propose a learning algorithm that optimizes CSB values in the frequency domain, and further extend the idea to optimizing CSB values in both the time and frequecy domains in [10].

When joint optimization is performed, it is often in a centralized entity. In [11], where ABS patterns and CSB values are jointly optimized and the surrounding macrocells of a picocell must offer ABSs on the same subframes. The algorithm has provable guarantees, and furthermore, it accounts for network topology, traffic load and the interference map for picocells and macrocells. The authors of [12] propose optimized ABS patterns and CSB values jointly and efficiently for large networks. System level simulation results illustrate significant gains from the optimization in both network capacity and data rate fairness. The collaborators Bedekar and Agrawal, in [13], simplify the joint ABS and CSB optimization problem in order to optimize $\mathrm{ABS}$ ratios and propose an iterative algorithm that successively improves the assignment of users to cells and the muting proportion, and prove its convergence to the optimal solution.

A distributed eICIC algorithm is proposed in various studies [14]-[18]. In [14], a distributed algorithm is proposed by Pang et al., where the number of ABS is determined without considering CSB values. The optimization is only done for static configurations using long-term statistics. The authors in [15] present a dynamic eICIC by adapting the ABS patterns in response to variations in network load. In [16], the operation of eICIC in a realistic deployment is proposed based on site-specific data from a dense urbanized European capital. In [17], the authors use moving average crossover (MAC) based on trading know-how to propose a new dynamic ABS eICIC algorithm, and through use of system-level simulations, attest that the proposed algorithm outperforms the best placed eICIC. Finally, the study in [18], which shows that dynamic ICIC can provide useful and stable performance gains in both homogeneous and heterogeneous networks. One also notes that the gains of dynamic ICIC are greater in heterogeneous networks of macrocells and picocells than in homogeneous networks of only macrocells.

Currently, several papers have been published with proposals for game theory based interference mitigation for LTE/LTE-A networks. Potential game based solutions for distributed eICIC optimization are considered in [7], [19]-[21].

Although ICIC is a subject that has been very well explored by academics and the telecommunications industry, the literature is lacking studies that explore improvements to eICIC schemes. Thus, this paper presents a performance comparison between the eICIC optimization, and a scenario with the optimized coordination technique, eICIC+.

\section{OVERVIEW ON EICIC IN LTE-A NETWORKS}

To deal with the interference problem in HetNets, such as cell overlap between macrocells and picocells, enhanced ICIC (eICIC) has made several improvements to overcome the limitations of previous ICIC schemes. However, its actual implementation is not clearly specified by the standard and there is no indication as to how to set the CSB and ABS parameters for different network configurations. In this section, we describe the main eICIC parameters.

\section{A. Almost blank subframe (ABS)}

The main change from static ICIC is the addition of time domain partitioning schemes based on time resource partitioning to limit interference to users on the outer edge of picocells. According to the work in [19], the specification of ABS defines a pattern of sub-frames during which a macrocell should mute and as such does not send any traffic channels. The ABS mechanism is illustrated in Fig. 1

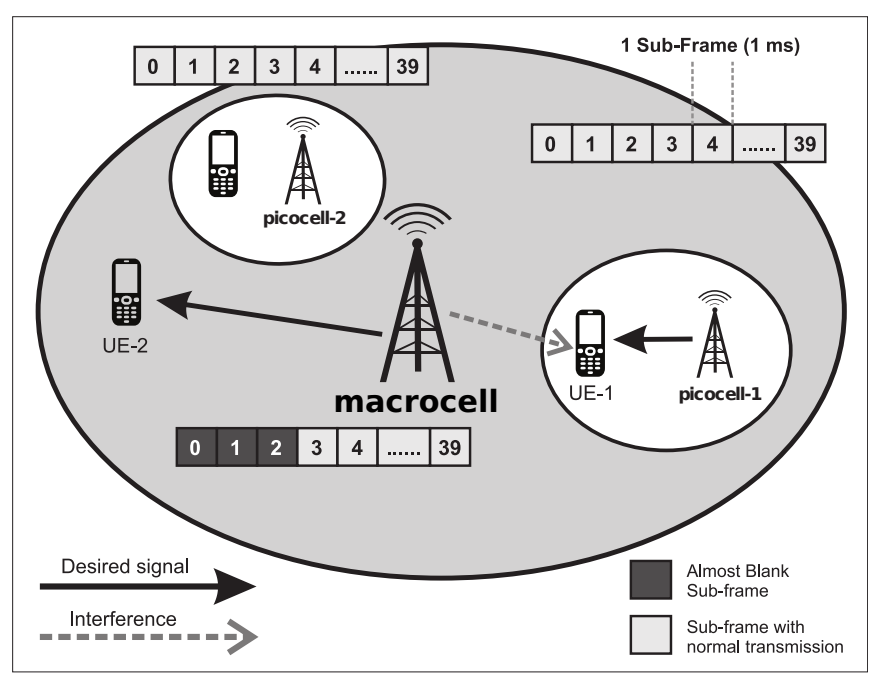

Fig. 1. ABS technique for interference in LTE-A HetNets. CIEEE [19]. 
This pattern is signaled in the form of a 40-subframe bitmap lasting $40 \mathrm{~ms}$. A neighboring picocell that possesses UEs interfered by the macrocell will preferentially schedule those UEs in the protected subframes. Other UEs located at the cell center can be scheduled over all subframes since the interference experienced from the macrocell aggressor is negligible compared to the signal of the picocell.

The UE-1, associated with picocell-1, suffers macrocell interference because it is located on the edge of a picocell. During each of the three subframes patterns of $40 \mathrm{~ms}$, the macrocell is muted and sends no data traffic. Interference is reduced during such $\mathrm{ABS}$; as picocell-1 will take advantage of the scheduled UE-1.

During ABS, macrocell does not transmit physical a downlink shared channel (PDSCH), creates "protected" subframes or "muting" for a picocell by reducing its transmit power, but for backward compatibility certain signals must be transmitted on all downlink subframes, even if muted [22].

The eICIC proposal in the LTE standards allows the timesharing of subframes to link transmissions between macrocells and picocells, in order to mitigate the high interference experienced especially by picocell downlink users. The notion of eICIC via $A B S$ is often coupled in the literature with another important technique for HetNets, namely that of appropriate resource allocation to ensure that picocells are not underused and not overloaded.

\section{B. Cell selection bias (CSB)}

An important approach for user association that has attracted a lot of interest lately is the concept of biased user association. This has been proposed by 3GPP in Release 10 [23], to extend the coverage of macrocells and increase their load. This mechanism is also called cell range extension (CRE). The strength of the power received by a user from a picocell would be artificially added by an offset, in order to offload macrocells and to ensure that more users attach to picocells. This offset is often named cell selection bias (CSB) [19]. The CSB mechanism is described in Fig. 2 .

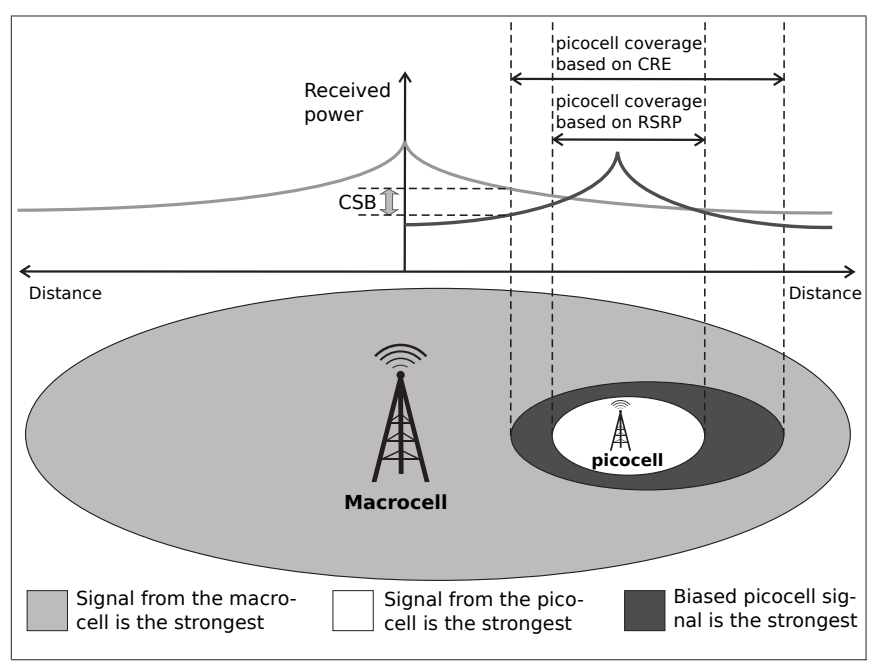

Fig. 2. CSB technique for interference in LTE-A HetNets. (C)IEEE [19].
Each two neighboring cells range from $(-24 d B)$ to $(24 d B)$ but reasonable values are in the $10 \mathrm{~dB}$ range. In general, some reciprocity is required in the CSB where CSB from macrocell1 to macrocell-2 is equal to minus CSB from macrocell-2 to macrocell-1. Playing with the offset, artificially biases the attraction of a neighboring cell, this can "force" the user to perform the handover from or towards this neighboring cell in an indirect manner. For instance, when CSB from a macrocell to a picocell increases, it implies that more users would be offloaded from the macrocell to the picocell and this enlarges the coverage of the macrocell [24].

The purpose of CRE is to offload more traffic from macrocells to picocells and hence achieve larger cell splitting gains. By adding a CSB, the service area of picocells increases and more users are offloaded to picocells. The need for hetnets interference management schemes stems from the fact that users in the picocell expansion area are more vulnerable to stronger interference signals than useful signals from the associated serving picocell [25].

\section{SYSTEM MODEL}

We consider a randomly generated HetNet that consists of macrocells and as such denote $\mathcal{M}$ and $\mathcal{P}$ as the set of all macrocells and picocells, respectively. Denote $\mathcal{M}_{c}$ and $\mathcal{P}_{c}$ as the macrocell and picocells in the center cluster of the HetNet, where the center cluster is surrounded by six clusters which are identical to the center cluster. We define that there is only one macrocell located at the center of each hexagon, and each hexagon has the same number of picocells. In addition, we adopt the system model adapted in [7]. Table I] summarizes the notation used in this paper.

Let $\mathcal{U}$ be the set of all users in the system. Denote $\mathcal{M}_{u}$ as the macrocell that is located in the same hexagon as user $u$. We assume that only the macrocells in the same hexagon or in the adjacent hexagons can serve a user. Moreover, let $\mathcal{U}_{i}$ be the set of users associated with station $i$, where $i \in \mathcal{M} \cup \mathcal{P}$. The vector $\lambda$ specifies the CSB values of all macrocells and picocells, where $\lambda_{i}$, the CSB value of station $i$, belongs to a predefined set $C$. Let $P_{i, u}^{R x}$, be the RSRP of user $u$ from station $i$, and the received power depends on the transmission power of the station, the distance between the station and the UE, and the loss due to shadow fading. Equation 1 gives the station that serves user $u$

$$
\epsilon(u, \lambda) \triangleq \underset{i \in \mathcal{M} \cup \mathcal{P}}{\operatorname{argmax}}\left(P_{i, u}^{R x}+\lambda_{i}\right)
$$

Let us assume there are $N_{S}$ subframes in the time domain and $N_{R}$ resource blocks (RBs) in the frequency domain. All subframes have the same duration, and all RBs are identical in terms of bandwidth. A PRB is formed by a pair of subframe and RB, and we denote $N_{B}:=N_{S} \cdot N_{R}$ as the total number of PRBs available at each macrocell. All macrocells transmit at a fixed power level on PRBs that correspond to non-ABSs, and all picocells transmit at a fixed power level on all PRBs.

In this paper, we assume that only the macrocells would offer ABS while the picocells always transmit on all subframes. Let $\alpha_{m}$ be the binary vector specifying the ABS pattern of 
macrocell $m$, where $\alpha_{m, j}=0$ means the $j$-th subframe of macrocell $m$ is an ABS and $\alpha_{m, k}=1$ means the $k$-th subframe of macrocell $m$ is a non-ABS, respectively. The signal to noise plus interference ratio (SINR) of a user $u$ on PRB $b$ when associated with macrocell $m$ can be calculated as

$$
\operatorname{SINR}_{u, b}^{m}=\frac{h_{u, b}^{m} P_{m, u}^{R x} \cdot \alpha_{m}(\tau(b))}{P_{\iota_{m}, u, b}^{I F}+N_{O} \mathcal{B}},
$$

where $h_{u, b}^{m}$ gives the fast fading gain on PRB $b$ from macrocell $m$ to user $u, \tau(b)$ returns the subframe index of PRB $b, \iota_{m}$ denotes the set of stations whose transmission will interfere with the users located in the same hexagon as macrocell $m$, $P_{\iota_{m}, u, b}^{I F}$ is the sum of interference user $u$ received from stations in $\iota_{m}$ at PRB $b, N_{0}$ denotes the additive white gaussian noise (AWGN) spectral density, and $\mathcal{B}$ is the bandwidth of a PRB. Similarly, the SINR of user $u$ on PRB $b$ when associated with picocell $p$ is given by

$$
\operatorname{SINR}_{u, b}^{p}=\frac{h_{u, b}^{p} P_{p, u}^{R x}}{P_{\iota_{p}, u, b}^{I F}+N_{O} \mathcal{B}} .
$$

The rate achieved by user $u$ at PRB $b$ is denoted as $r_{u, b}$, where $b \in\left[1, N_{B}\right]$. Due to the small-scale fading and potentially different ABS patterns of the interfering macrocells, a user can obtain different throughput on different PRBs and the rate achieved is calculated by Shannon's capacity formula, i.e.,

$$
r_{u, b}= \begin{cases}\mathcal{B} \cdot \log _{2}\left(1+\operatorname{SINR}_{u, b}^{m}\right), & m \in \mathcal{M}, \\ \mathcal{B} \cdot \log _{2}\left(1+\operatorname{SINR}_{u, b}^{p}\right), & p \in \mathcal{P} .\end{cases}
$$

Let $x_{u, b}$ be a binary variable indicating whether PRB $b$ is allocated to user $u$ by its serving station, where $x_{u, b}=1$ means that PRB $b$ is allocated to user $u$ and $x_{u, b}=0$ means otherwise. Denote $w_{u}$ as the positive weighting factor of user $u$ such that the each user has a throughput utility function, but no minimum rate is guaranteed, which can be interpreted as best effort user. We formulate the eICIC optimization problem as follows

$$
\begin{aligned}
\operatorname{maximize} & \sum_{i \in \mathcal{M} \cup \mathcal{P}} \sum_{u \in \mathcal{U}_{i}} w_{u} \cdot \ln \sum_{b=1}^{N_{B}} x_{u, b} \cdot r_{u, b}, \\
\text { subject to } & \sum_{u \in \mathcal{U}_{m}} x_{u, b}=\alpha_{m}(\tau(b)), \\
& \forall m \in \mathcal{M}_{c}, b \in\left[1, N_{B}\right], \alpha_{m} \in A, \\
& \sum_{u \in \mathcal{U}_{p}} x_{u, b}=1, \forall p \in \mathcal{P}_{c}, b \in\left[1, N_{B}\right], \\
& x_{u, b} \in\{0,1\}, \forall u \in \mathcal{U}, b \in\left[1, N_{B}\right], \\
& \lambda(s) \in C, \forall s \in \mathcal{P}_{c},
\end{aligned}
$$

\begin{tabular}{|c|c|}
\hline Notation & Description \\
\hline$A$ & $\begin{array}{l}\text { Set of vectors from which macrocells can choose ABS } \\
\text { pattern }\end{array}$ \\
\hline $\mathcal{B}$ & Bandwidth per RB \\
\hline$C$ & Set of CSB values from which a picocell can choose from \\
\hline$\epsilon(u, \lambda)$ & The macrocell that user $u$ is associated with \\
\hline$h_{u, b}^{i}$ & Fading gain from station $i$ to user $u$ at PRB $b$ \\
\hline $\mathcal{M}$ & Set of all macrocells \\
\hline $\mathcal{M}_{c}$ & Set of macrocells in the center cluster \\
\hline $\mathcal{M}_{u}$ & $\begin{array}{l}\text { Set of macrocells that is located in the same hexagon as } \\
\text { user } u\end{array}$ \\
\hline$N_{0}$ & Noise power spectral density \\
\hline$N_{B}$ & Number of PRB \\
\hline$N_{R}$ & Number of RB (in frequency domain) \\
\hline$N_{S}$ & Number of subframes (in time domain) \\
\hline $\mathcal{N}_{i}^{A t t}$ & $\begin{array}{l}\text { Set of stations whose } i \text { user attachment can be affected } \\
\text { by the change of CSB value of station } i\end{array}$ \\
\hline $\mathcal{N}_{i}^{I F}$ & $\begin{array}{l}\text { is the set of stations whose attached users can be inter- } \\
\text { fered by the transmissions of station } i\end{array}$ \\
\hline $\mathscr{P}$ & Set of all picocells \\
\hline $\mathcal{P}_{c}$ & Set of picocells in the center cluster \\
\hline$P_{i, u}^{R x}$ & The RSRP of user $u$ from station $i$ \\
\hline$P_{\iota_{m}, u, b}^{I F}$ & $\begin{array}{l}\text { The sum of interference at user } u \text { received from stations } \\
\text { in } \iota_{m} \text { at PRB } b\end{array}$ \\
\hline$r_{u, b}$ & Achieved rate of user $u$ at PRB $b$ \\
\hline$\tau(b)$ & The index of subframe of PRB $(b)$ \\
\hline $\mathcal{S}_{i}$ & Set of the strategy of player $i$ \\
\hline $\mathcal{U}$ & Set of all users in the system \\
\hline $\mathcal{U}_{i}$ & $\begin{array}{l}\text { Set of users associated with macrocells in set } i \text { or with } \\
\text { macrocell } i \text { in the system }\end{array}$ \\
\hline$x_{u, b}$ & $\begin{array}{l}\text { Indicator of whether user } u \text { occupies PRB } b \text { of the } \\
\text { serving station }\end{array}$ \\
\hline$w_{u}$ & Weighting factor of UE $u$ \\
\hline$\alpha_{m}$ & ABS pattern of macrocell $m$ \\
\hline$\lambda$ & Vector specifying CSB values of all macrocells \\
\hline$\lambda_{i}$ & Vector specifying CSB values of station $i$ \\
\hline$\iota_{m}$ & $\begin{array}{l}\text { The set of stations whose transmission will interfere with } \\
\text { the users located in the same hexagon as macrocell } m\end{array}$ \\
\hline $\boldsymbol{\beta}$ & Weight of metric $J$ \\
\hline$\gamma$ & Weight of metric $J$ \\
\hline
\end{tabular}

where $5 \mathrm{~b}$ specifies that a macrocell can adopt one of the ABS patterns in $A$ and only non-ABS PRBs can be assigned to the users such that at most one user can occupy a PRB, 5c states that all PRBs from picocells can be allocated to the users and at most one user can occupy a PRB, and $5 \mathrm{e}$ means that a picocell can adopt one of the CSB values specified in $C$.
TABLE I

SUMMARY OF NOTATION

\section{Distributed Solution For EICIC Optimization}

In this section, the authors herein aim at developing a distributed solution to the problem described in Section IV by formulating an exact potential game based on the algorithm described in paper[7]. In addition, we present the downlink schedulers $\Phi$ used in this paper.

\section{A. Exact Potential Game Formulation}

Potential games were defined and their properties discussed in [26]. A game is called an exact potential game if there exists an exact potential function such that the change in the value of the exact potential function due to the change of a player's strategy is the same as the change of the player's payoff. The key to the exact potential game formulation is found in the appropriate definition of the payoff function.

A finite game consists of a finite set of players, a finite set of strategies of each player, and the payoff functions of the players, where the payoff of a player is a function of the strategies played by all the players. A strategy profile gives the strategies adopted by all the players, and a Nash equilibrium can be achieved if players take turns randomly and play their 
best responses or better responses [27], where it is understood that all other players fix their strategies.

The best response dynamics solves an exact potential game by iteratively finding the strategies that maximize the payoff functions of the players selected in each iteration [6]. We provide a distributed algorithm that jointly optimizes the ABS patterns and CSB values in HetNets. We formulate the problem as an exact potential game that allow us to solve them distributively using simple algorithms based on best/better response dynamics.

Using the approach in [7], let the stations be the players, so that $\mathcal{M} \cup \mathcal{P}$ defines the set of player. Each player $i$ chooses a strategy $s_{i}$ from a set of strategies $\mathcal{S}_{i}$. The underlying assumption is that ABS is allowed in macrocells but not in picocells, and all stations can set their CSB values according to $C$. Let vector s specify the strategies of all players, where $s(i)$ gives the strategy of station $i$. Define the strategies selected by all players except player $i$

$$
\mathbf{s}_{-i} \triangleq\left(s_{1}, \ldots, s_{i-1}, s_{i+1}, \ldots, s_{|\mathcal{M}|+|\mathcal{P}|}\right)
$$

as the strategies of all players other than player $i$. Denote

$$
\left(s_{i}^{\prime}, \mathbf{s}_{-i}\right) \triangleq\left(s_{1}, \ldots, s_{i-1}, s_{i}^{\prime}, s_{i+1}, \ldots, s_{|\mathcal{M}|+|\mathcal{P}|}\right)
$$

so that player $i$ adopts strategy $s_{i}^{\prime}$ and the strategies of the remaining players are specified by $s_{-i}$.

Let $\mathcal{N}_{i} \triangleq\{i\} \cup \mathcal{N}_{i}^{I F} \cup \mathcal{N}_{i}^{A t t}$, where $i \in \mathcal{M} \cup \mathcal{P}, \mathcal{N}_{i}^{I F}$ is the set of stations whose attached users can be inhibited by the transmissions of station $i, \mathcal{N}_{i}^{A t t}$ is the set of stations whose $i$ user attachment can be affected by the change of CSB value of station $i$. Let the utility of station $i \in \mathcal{M} \cup \mathcal{P}$ be

$$
U_{i}(\mathbf{s}) \triangleq \sum_{u \in \mathcal{U}_{i}} w_{u} \cdot \ln \sum_{b=1}^{N_{B}}\left(x_{u, b}\right) \cdot r_{u, b}
$$

where $x_{u, b}$ is obtained by some scheduling scheme given $s$ so that the constraint in 5b, 5c and 5d are satisfied. The aggregate utility of all the players is given as

$$
U(\mathbf{s})=\sum_{i \in \mathcal{M} \cup \mathcal{P}} U_{i}(\mathbf{s})
$$

and the payoff function of player $i$ is defined as

$$
V_{i}(\mathbf{s}) \triangleq \sum_{j \in \mathcal{N}_{i}} U_{j}(\mathbf{s})
$$

In the following theorem, we show that when $N_{i}^{I F}=i \cup$ $N(i, 1), U(s)$ is an exact potential function.

Theorem 1. If $N_{i}^{I F}=i \cup N(i, 1)$, then $U(\cdot)$ is an exact potential function of the game $\Gamma$, so that $\Gamma$ is an exact potential game.

Proof. See [7].

\section{B. Proposal Improvements eICIC Optimization framework}

In this paper, the objective is to prioritize the traffic of users located at the edge of the cell, which are those that face the highest levels of interference. We formulate the optimization problem as a game where, in order to maximize a modified utility function aiming at improvements on eICIC. This strategy exploits the diversity of users across the network in the frequency and time domain.

We adapt the game theoretic frameworks in [7], derived from Equation 11, as well as propose some improvements on these algorithms in order to achieve better performance, called eICIC+ and formulate the following problem

$$
\text { maximize } \sum_{i \in \mathcal{M} \cup \mathcal{P}} \sum_{u \in \mathcal{U}_{i}} w_{u} \cdot \ln \sum_{b=1}^{N_{B}} x_{u, b} \cdot r_{u, b},
$$

The proposed algorithm eICIC + finds the right balance between fairness and throughput by adjusting certain variables in the metric [28]. The utility function considers the achieved throughput and instantaneous user data rates to increase the likelihood of allocation and to serve users according to priority requirements and thus achieve better network performance. In this sense, the option chosen to improve eICIC optimization performance was to change its metric to maintain its basic characteristics, for which the metric of eICIC+ optimization is presented below

$$
\begin{aligned}
& \operatorname{maximize} \sum_{i \in \mathcal{M} \cup \mathcal{P}} \sum_{u \in \mathcal{U}_{i}} w_{u} \cdot \ln \sum_{b=1}^{N_{B}} x_{u, b} \cdot J \text {, } \\
& \text { subject to } \mathrm{J}=\frac{\left[r_{u, b}\right]^{\beta}}{\left[\bar{r}_{u}(t)\right]^{\gamma}} \text {. }
\end{aligned}
$$

Different realizations of $\beta$ and $\gamma$ will affect the elements in average data rate and instantaneous data rate. Thus it is seen that the eICIC+ optimization seeks an adaptation both to deliver fairness to users as well as high transfer rates (e.g., see [28]).

The higher the $\beta$ values, greater will be the achievable influence of the instantaneous data rate, thus improving the likelihood of serving a user with good channel conditions. This translates into a higher throughput on the network, but with a lower fairness index. As one increases the metric, one seeks to broaden the influence of the flow rate achieved at previous times, this increases the chance that a user who has reached lower throughput values will be scheduled, resulting in a low throughput of the network, but a high level of fairness can be predicted.

Now, on the other hand, when the $\gamma$ value is increased, the user-equal rate escalation scenario is achieved, thus delivering the same rate to all requesters. Thus, the algorithm metric is responsible for modifying the impact of the resource allocation policy on the expected instantaneous data rate for UEs on the network, so that with an equal rate for all users, the algorithm allocates resources for UEs such that they achieve an equal transfer rate.

Algorithm 1 provides more details of the eICIC+ optimization. Therefore the proposed eICIC + optimization algorithm based on exact potential game formulation [7] is given as follows: 


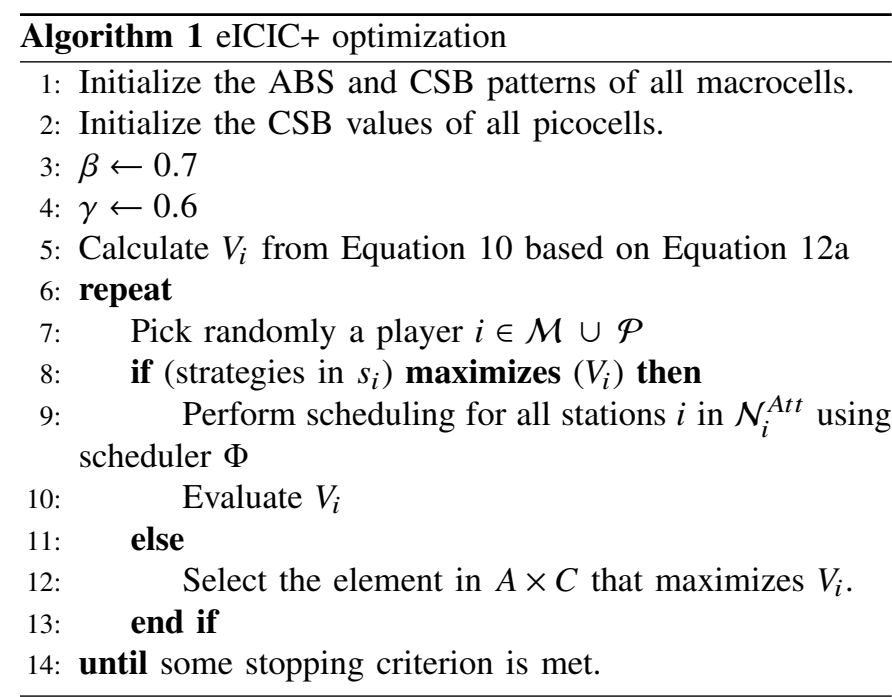

\section{Proportional Fair (PF) Scheduler}

The PF tries to strike a balance between spectral efficiency and fairness by adjusting the scaling rate according to the instantaneous channel quality in relation to its performance over time [29]. For station $s$, the $b$-th PRB at subframe $\tau(b)$, given that the subframe is not an ABS, will be allocated to the following user

$$
\widehat{u}_{b} \triangleq \underset{u \in \mathcal{U}_{i}}{\operatorname{argmax}} \frac{r_{u, b}}{\bar{r}_{u}(\tau(b))},
$$

where $\tau(b)$ gives the subframe index of the b-th PRB, $b \in$ $[1, B]$, and $\bar{r}_{u}(\tau(b))$ is the long-term average throughput of user $u$ in subframe $\tau(b)$ which is calculated as

$\bar{r}_{u}(t)=\left(1-\frac{1}{s_{w}}\right) \bar{r}_{u}(t-1)+\frac{1}{s_{w}} \sum_{\{b \mid \tau(b)=t\}} r_{u, b} \cdot \mathcal{I}\left\{\widehat{u}_{b}=u\right\}$.

In the equation above, $s_{w}$ is the size window which is a design parameter and $\mathcal{I}\{\cdot\}$ is the indicator function.

The goal is to use the long-term average throughput $\bar{r}_{u}(t)$ to act as a data rate weighting factor $r_{u, b}$, so that users of channels with a low service quality can be attended to within the given period of time.

\section{Round-Robin (RR) Scheduler}

It is probably the simplest scheduler found in the literature [30]. It assigns resource block group (RBGs) sequentially to UEs without any concern for channel conditions, thereby, dividing available resources among active streams. If the number of RBGs is greater than the number of active streams, all streams can be allocated to the same subframe. In the next subframe, allocation will start from the last unallocated stream.

In scheduling, time resources are evenly divided among users one after the other and without any priority, scheduling is based on the availability of RBs during the scheduling process. The metric is similar to that of FIFO scheduling, the difference is in $T_{u}$, which is the last access to user $u$ broadcast channel.

$$
\widehat{u}_{b} \triangleq \underset{u \in \mathcal{U}_{i}}{\operatorname{argmax}} t-T_{u}
$$

\section{Vi. Performance Evaluation}

In order to evaluate the performance of the eICIC optimization, a simulation environment in the MATLAB software was modeled with reference to [6]. Table [I] presents the main simulation parameters which are based on the paper described in [31].

TABLE II

SimULATION PARAMETERS

\begin{tabular}{ll}
\hline Parameter & Value \\
\hline Antenna gain & $14 \mathrm{dBi}$ \\
Antenna per site & Omnidirectional $x 1$ \\
Bandwidth & $5 \mathrm{MHz}$ \\
Cell geometry & Hexagonal \\
CSB values & $C:=\{0,3,6,9,12,15\} \mathrm{dB}$ \\
Dist. min. of macrocell per user & $35 \mathrm{~m}$ \\
Dist. min. of macrocell to picocell & $75 \mathrm{~m}$ \\
Dist. min. of picocell per user & $10 \mathrm{~m}$ \\
eICIC $+(\boldsymbol{\beta})$ & 0.7 \\
eICIC $+(\boldsymbol{\gamma})$ & 0.6 \\
Inter macrocells distance & $1000 \mathrm{~m}$ \\
Macrocell maximum power $\left(P_{t}\right)$ & $40 \mathrm{~W}$ \\
Number of RBs & 25 \\
Number of sites & 7 \\
Path loss from macrocell to user & $128.1+37.6 \log _{10} d, d$ in km \\
Path loss from picocell to user & $140.7+36.7 \log _{10} d, d$ in $\mathrm{km}$ \\
Penetration loss (PenL) & $10 \mathrm{~dB}$ \\
Picocell maximum power $\left(P_{t}\right)$ & $1 \mathrm{~W}$ \\
RB bandwidth & $180 \mathrm{kHz}$ \\
Schedulers & Proportional Fair \\
Shadow fading $(\zeta)$ & Round-Robin \\
Subcarrier frequency & Log-normal distribution \\
Thermal noise density & $15 \mathrm{kHz}$ \\
Traffic model & $-174 \mathrm{dBm} / \mathrm{Hz}$ \\
TTI & Full buffer \\
\hline
\end{tabular}

The geometry of the cell for our simulated LTE system is hexagonal. The scenario consists of 7 clusters consisting of macrocells and picocells, six adjacent to the central cluster. The six neighboring clusters of the central cluster are exact copies of the central cluster responsible for creating the interference scenario. The inter-macrocells distance is $1000 \mathrm{~m}$, which corresponds to an LTE network deployed in an urban area. In each cell, $25 \mathrm{RBs}$ are available as the operational bandwidth is $5 \mathrm{MHz}$. However, the traffic model is full buffer, meaning all available RBs are permanently allocated to the active UEs in the network.

Simulation studies are performed on eICIC optimization by randomly generating 10 HetNets topologies and then the central cluster average of the performance measures of each topology. It is assumed that there is only one macrocell located in the center of each hexagon, and each hexagon has the same number of picocells. In the central cluster of each topology, a number of picocells (ranging from one to five), where the picocells are placed randomly. In addition, for each hexagon in the center cluster, six users are placed within 100 meters from each picocell and another four users are placed within the hexagon. The distribution of the distances from the six users who are intentionally placed within 100 meters of a picocell, and the directions from the picocell to the six users are random. The other four users are similarly generated except the reference point, which the respective macrocell. 
It is assumed that users are static. In addition, each PRB experiences Rayleigh fading with variance 1 . The shadow fading of a macrocell for a user is calculated by first adding a common shading value and a random shading value and dividing the sum by $\sqrt{2}$, where both shading values are generated according to the log normal distribution. The weighting factors of all users are set be 1 , i.e., $w_{u}=1$ for all $u$. $N_{S}$ is set be 10 and $N_{R}$ is set to 3 . In all simulation results, we compared a technique that uses an exact potential game (named eICIC in the graphics), the proposed eICIC+ optimization and a scenario without interference control, NULL.

Spectral efficiency (SE) is defined as the average of bits transmitted per second per $\mathrm{Hz}(\mathrm{bps} / \mathrm{Hz})$ of all allocated PRBs. The obtained SE results are shown in Fig. 3 and Fig. 4 , considering the scheduling algorithms $\mathrm{PF}$ and $\mathrm{RR}$, respectively. Noteworthy here is that with the increase of picocells, there is an improvement of SE when using the eICIC and eICIC+ techniques compared to the scenarios without interference control. Thus, such techniques proved to be important in terms of SE for the simulated scenario, that is, in HetNets with a higher densification level. In addition, it is noteworthy that the SE improvement was more significant in the scenario with the use of the PF scheduling algorithm.

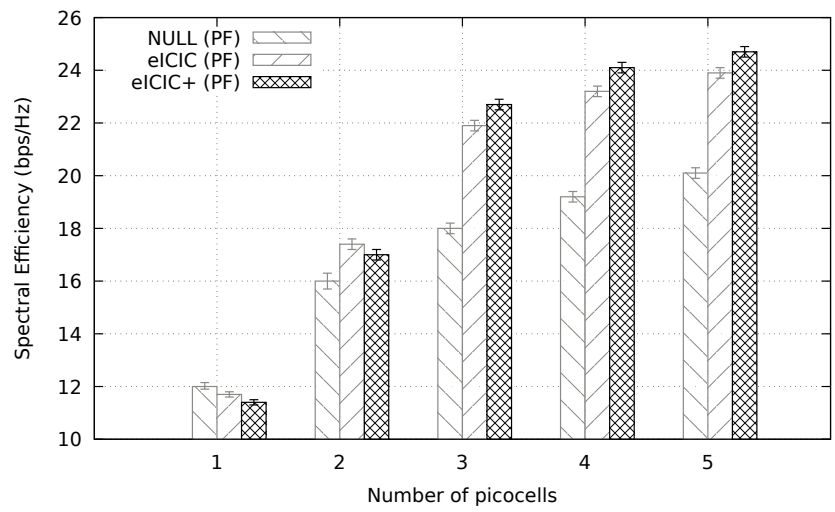

Fig. 3. Comparative index of SE as a function of the number of picocells using the PF algorithm.

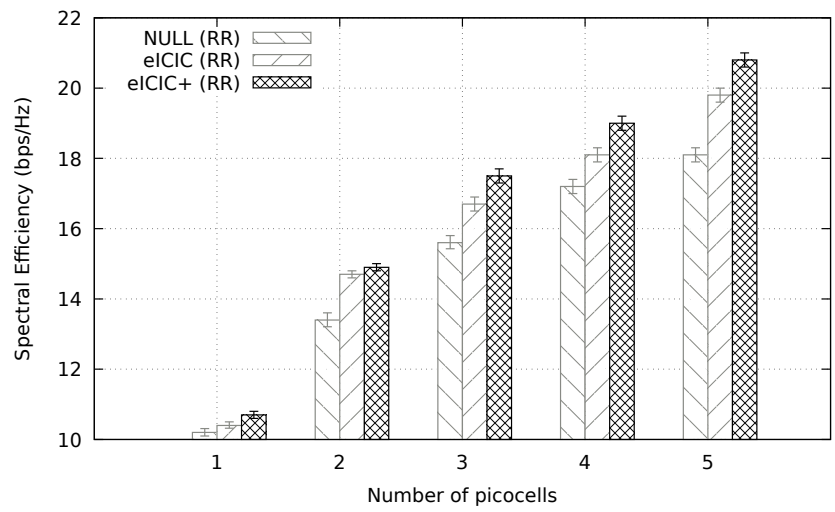

Fig. 4. Comparative index of $\mathrm{SE}$ as a function of the number of picocells using the RR algorithm.

The energy efficiency (EE) calculated as the number of transmitted bits divided by the transmission power is presented in Fig. 5 and Fig. 6, considering the PF and RR scheduling algorithms, respectively. The use of eICIC and eICIC+ techniques resulted in an increase of EE in all scenarios with the exception of the one picocell scenario in conjunction with the use of the PF algorithm. In general, it is noted that the eICIC+ strategy had better results than the eICIC technique, proving to be a more appropriate technique for the coordination of interferences in the analyzed scenarios, especially with the increase in the number of picocells.

Since network densification is a key element in increasing system coverage and meeting in growing demand, the use of interference coordination techniques, particularly eICIC+, has enabled improvements in spectral and energy efficiency and are, therefore, important in expanding the capacity of the network.

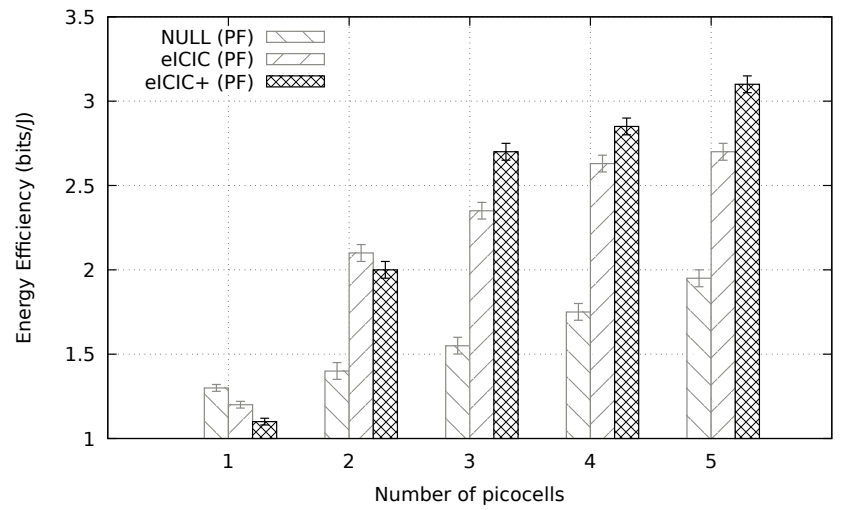

Fig. 5. Comparative index of $\mathrm{EE}$ as a function of the number of picocells using the PF algorithm.

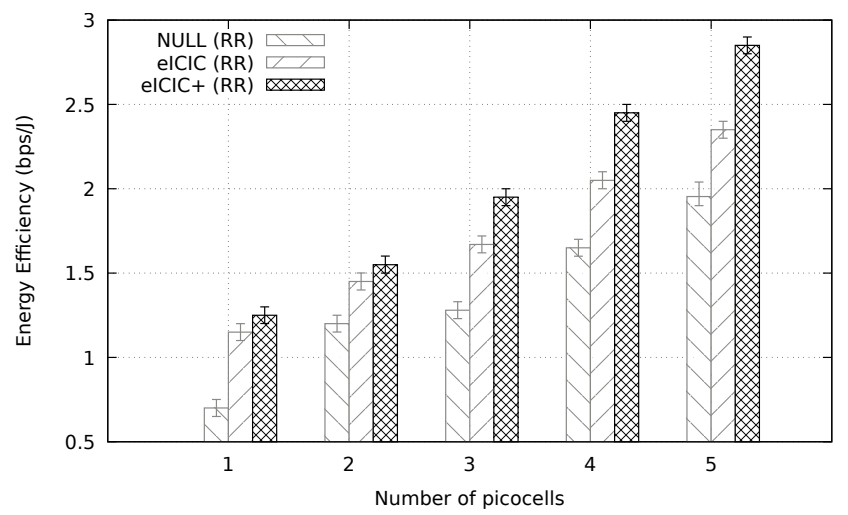

Fig. 6. Comparative index of $\mathrm{EE}$ as a function of the number of picocells using the RR algorithm.

The throughput of the worst $5 \%$ of rates achieved by users that tend to have the highest levels of interference is analyzed, that is, they are at the edge of the cell in the central cluster. Fig. 7 and Fig. 8 show the cumulative distribution function (CDF) of the said rate, considering the PF and RR scheduling algorithms, respectively, and each hexagon has five picocells. Through this configuration, eICIC optimization can improve the median value of the worst $5 \%$ users' achieved rates of 
about $10.89 \%(7.65 \%)$ and eICIC+ optimization can further improve the median value by about $16.54 \%$ (12\%) considering the PF (RR) algorithm.

As a result, when mobile operators seek to improve throughput of the user edge cell, eICIC+ optimization in conjunction with the PF algorithm proves itself to be the most appropriate technique for these scenarios.

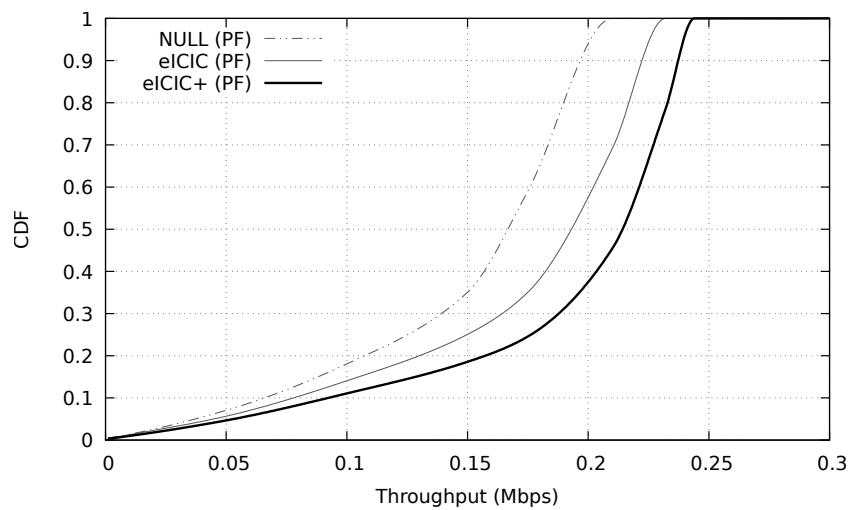

Fig. 7. The cumulative distribution function (CDF) of the worst $5 \%$ users' achieved rates in the center cluster, where the PF scheduler is used and each hexagon has five picocells.

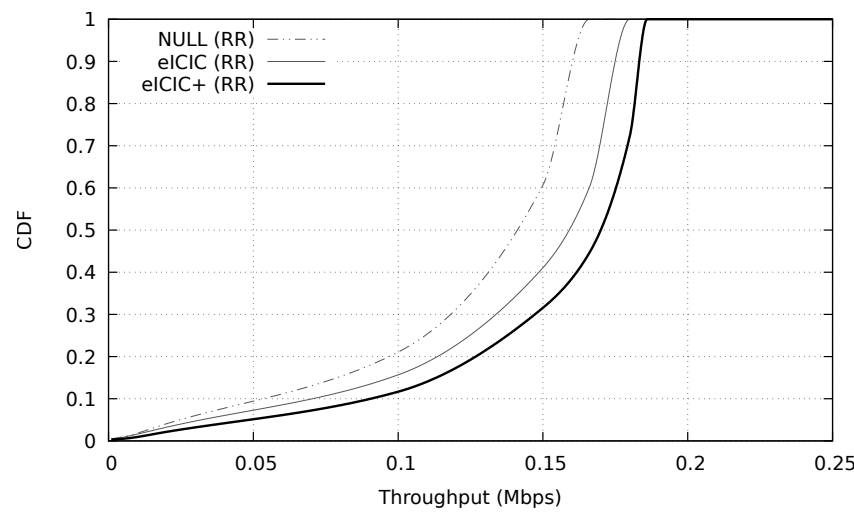

Fig. 8. The cumulative distribution function (CDF) of the worst $5 \%$ users' achieved rates in the center cluster, where the RR scheduler is used and each hexagon has five picocells.

For other quantities of picocells, the improvements percentages range of the worst $5 \%$ users' achieved rates compared to the non-optimization case are shown on Table III]

It is important to note that in the one picocell scenario, although the eICIC+ scheme did not always achieve better SE and EE results than eICIC, the worst 5\% users' achieved rates increased considerably for both scheduling algorithms. In addition, for the other quantities of picocells one also noted for the eICIC+ scheme an increase in the throughput of the worst 5\% users' achieved rates, keeping the SE and EE higher than the eICIC scheme, especially when using the PF scheduling algorithm.

Another noteworthy point is that the use of the PF scheduling algorithm has always presented superior results for the statistics presented so far, in relation to the RR algorithm in the analyzed scenarios. Given this fact, it is noteworthy
TABLE III

Percentage OF THE WORST 5\% USERS' ACHIEVED Rates

\begin{tabular}{|c|c|c|c|}
\hline Picocells & Schedulers & $\begin{array}{c}\text { Improvement (\%) } \\
\text { eICIC }\end{array}$ & $\begin{array}{c}\text { Improvement (\%) } \\
\text { eICIC+ }\end{array}$ \\
\hline \multirow{2}{*}{1} & PF & $30 \%$ & $44 \%$ \\
\cline { 2 - 4 } & RR & $13.05 \%$ & $19.04 \%$ \\
\hline \multirow{2}{*}{2} & PF & $22.22 \%$ & $25.92 \%$ \\
\cline { 2 - 4 } & RR & $11.90 \%$ & $17.87 \%$ \\
\hline \multirow{2}{*}{3} & PF & $17.63 \%$ & $21.31 \%$ \\
\cline { 2 - 4 } & RR & $11.88 \%$ & $16 \%$ \\
\hline \multirow{2}{*}{4} & PF & $13.44 \%$ & $19.03 \%$ \\
\cline { 2 - 4 } & RR & $8.65 \%$ & $14.77 \%$ \\
\hline \multirow{2}{*}{5} & PF & $10.89 \%$ & $16.54 \%$ \\
\cline { 2 - 4 } & RR & $7.65 \%$ & $12 \%$ \\
\hline
\end{tabular}

that from two picocells the percentage of improvement of the worst $5 \%$ users' achieved rates, promoted by the eICIC+ scheme, when compared to eICIC, grew as the number of picocells increased. Thus, as the throughput of users with the highest levels of interference in the cell is a major concern of interference coordination schemes, the use of the eICIC+ scheme in conjunction with the PF algorithm, based on the results obtained, is a promising setting.

Table IV] summarizes some key performance indicators of various optimization scenarios. We note here that the ABS and CSB optimization presented a growth on the mean achieved user rate, when compared to the case where no optimization is performed, where a user's achieved rate is calculated by adding the capacity of each PRB that is allocated to that user. This increase is intensified by the use of eICIC and especially eICIC+.

TABLE IV

FAIRNESS INDICES AND AVARAGE ACHIEVED USER RATE

\begin{tabular}{|c|c|c|c|}
\hline Optimization & Schedulers & $\begin{array}{c}\text { Mean UE Rate } \\
\text { (bits/s/Hz) }\end{array}$ & Jain's Index \\
\hline \multirow{2}{*}{ eICIC+ } & PF & 196.02 & 0.6543 \\
\cline { 2 - 4 } & RR & 159.77 & 0.6891 \\
\hline \multirow{2}{*}{ eICIC } & PF & 189.86 & 0.6174 \\
\cline { 2 - 4 } & RR & 151.54 & 0.6263 \\
\hline \multirow{2}{*}{ ABS } & PF & 181.89 & 0.5646 \\
\cline { 2 - 4 } & RR & 149.68 & 0.5863 \\
\hline \multirow{2}{*}{ CSB } & PF & 172.89 & 0.5376 \\
\cline { 2 - 4 } & RR & 129.02 & 0.5431 \\
\hline \multirow{2}{*}{ No optimization } & PF & 168.07 & 0.5123 \\
\cline { 2 - 4 } & RR & 121.22 & 0.5203 \\
\hline
\end{tabular}

With respect to the scheduling algorithms, one notes that the increase in the average rate obtained by employing the PF algorithm resulted in a reduction of the fairness index when compared to the RR algorithm.

In addition, we analyzed variations of the $\beta$ and $\gamma$ parameters used in eICIC+ optimization, according Table $\mathrm{V}$. As a result, it was found that with the increase of $\beta$ (0.7 to 0.75) there is a downward trend in the justice index and an increase in the UE average rate. On the other hand, with the increase of $\gamma(0.6$ to 0.7$)$, a behavior contrary to the previous scenario was observed. These parameters can therefore be adjusted when one of the performance measures is to be optimized. 
TABLE V

FAIRNESS INDICES AND AVARAGE ACHIEVED USER RATE

\begin{tabular}{|c|c|c|c|c|}
\hline \multirow{2}{*}{ Parameters } & \multirow{2}{*}{ Schedulers } & \multicolumn{3}{|c|}{ Optimization } \\
\cline { 3 - 5 } & & \multirow{2}{*}{ eICIC+ } & eICIC+ & eICIC+ \\
\cline { 3 - 5 } & & & $\boldsymbol{\beta}=0.75$ & $\boldsymbol{\gamma}=0.7$ \\
\hline \multirow{2}{*}{ Jain's } & PF & 0.6543 & 0.6233 & 0.6577 \\
\cline { 2 - 5 } & RR & 0.6891 & 0.6369 & 0.6899 \\
\hline \multirow{2}{*}{ Mean UE } & PF & 196.02 & 196.74 & 195.04 \\
\cline { 2 - 5 } & RR & 159.77 & 160.02 & 158.22 \\
\hline
\end{tabular}

\section{CONCLUSION}

This paper proposes a study and improvement of the eICIC optimization in downlink in LTE-A Hetnets. A performance comparison is presented between the eICIC coordination method presented in 3GPP Release-10, and a scenario with the optimized interference coordination proposal, eICIC+. Simulation results showed that eICIC+ optimization does in fact improve spectral efficiency and energy efficiency, especially in scenarios of higher grid densification. In addition, eICIC+ can generate significant gains in the throughput of users located at the edge of the cell, who face the highest levels of interference. The integration of the modified eICIC+ optimization with the PF scheduling algorithm proved to be a good option in the scenarios analyzed.

As future work, we highlight the need to study the integration of other scheduling algorithms with the eICIC+ technique and interaction with the $\beta$ and $\gamma$ parameters in order to optimize performance on a HetNet.

\section{REFERENCES}

[1] Cisco, "Cisco visual networking index: Global mobile data traffic forecast update, 2016-2021, White Paper," Cisco, San Jose, CA, USA, Rep., 2018.

[2] H. Holma and A. Toskala, LTE Advanced: 3GPP Solution for IMTAdvanced. New York, NY, USA: Wiley, 2012.

[3] H. S. Dhillon, R. K. Ganti, F. Baccelli, and J. G. Andrews, "Modeling and analysis of k-tier downlink heterogeneous cellular networks," IEEE Journal on Selected Areas in Communications, vol. 30, no. 3, pp. 550560, April 2012. doi: 10.1109/JSAC.2012.120405

[4] 3GPP, "3GPP TR 36814 V9.0.0 Further Advancements for E-UTRA Physical Layer Aspects (Release 9)," p. 104, 2010.

[5] — "LTE; Evolved Universal Terrestrial Radio Access (E-UTRA) and Evolved Universal Terrestrial Radio Access Network (E-UTRAN); Overall description; Stage 2," p. 104, 2013.

[6] Y. Liu, C. S. Chen, C. W. Sung, and C. Singh, "A game theoretic distributed algorithm for feicic optimization in lte-a hetnets," IEEE/ACM Transactions on Networking, vol. 25, no. 6, pp. 3500-3513, Dec 2017. doi: 10.1109/TNET.2017.2748567

[7] Y. Liu, C. S. Chen, and C. W. Sung, "Joint optimization on inter-cell interference management and user attachment in lte-a hetnets," in 2015 13th International Symposium on Modeling and Optimization in Mobile, Ad Hoc, and Wireless Networks (WiOpt), May 2015, pp. 62-69. doi: 10.1109/WIOPT.2015.7151054

[8] A. Tall, Z. Altman, and E. Altman, "Self Organizing strategies for enhanced ICIC (eICIC)," arXiv e-prints, p. arXiv:1401.2369, Jan 2014. doi: 10.1109/WIOPT.2014.6850315

[9] M. Simsek, M. Bennis, and I. Güuvenc, "Enhanced intercell interference coordination in hetnets: Single vs. multiflow approach," in 2013 IEEE Globecom Workshops (GC Wkshps), Dec 2013, pp. 725-729. doi: 10.1109/GLOCOMW.2013.6825074

[10] M. Simsek, M. Bennis, and I. Guvenc, "Learning based frequencyand time-domain inter-cell interference coordination in hetnets," IEEE Transactions on Vehicular Technology, vol. 64, no. 10, pp. 4589-4602, Oct 2015. doi: 10.1109/TVT.2014.2374237
[11] S. Deb, P. Monogioudis, J. Miernik, and J. P. Seymour, "Algorithms for enhanced inter-cell interference coordination (eicic) in lte hetnets," IEEE/ACM Transactions on Networking, vol. 22, no. 1, pp. 137-150, Feb 2014. doi: 10.1109/TNET.2013.2246820

[12] F. Alfarhan, R. Lerbour, and Y. Le Helloco, "An optimization framework for lte eicic and reduced power eicic," in 2015 IEEE Global Communications Conference (GLOBECOM), Dec 2015, pp. 1-6. doi: 10.1109/GLOCOM.2015.7417601

[13] A. Bedekar and R. Agrawal, "Optimal muting and load balancing for eicic," in 2013 11th International Symposium and Workshops on Modeling and Optimization in Mobile, Ad Hoc and Wireless Networks (WiOpt), May 2013, pp. 280-287.

[14] J. Pang, J. Wang, D. Wang, G. Shen, Q. Jiang, and J. Liu, "Optimized time-domain resource partitioning for enhanced inter-cell interference coordination in heterogeneous networks," in 2012 IEEE Wireless Communications and Networking Conference (WCNC), April 2012, pp 1613-1617. doi: 10.1109/WCNC.2012.6214040

[15] S. Vasudevan, R. N. Pupala, and K. Sivanesan, "Dynamic eicic - a proactive strategy for improving spectral efficiencies of heterogeneous lte cellular networks by leveraging user mobility and traffic dynamics," IEEE Transactions on Wireless Communications, vol. 12, no. 10, pp. 4956-4969, October 2013. doi: 10.1109/TWC.2013.081413.121651

[16] K. I. Pedersen, B. Soret, S. Barcos, G. Pocovi, and H. Wang, "Study of dynamic eicic in a realistic urban deployment," in 2015 IEEE 81st Vehicular Technology Conference (VTC Spring), May 2015, pp. 1-5. doi: 10.1109/VTCSpring.2015.7146112

[17] Y. Lima de Melo, V. Junior, and T. Maciel, "Dynamic e-icic using moving average crossover," Journal of Communication and Information Systems, vol. 34, no. 1, pp. 87-91, Mar. 2019. doi: 10.14209/jcis.2019.9

[18] Z. Xiong, M. Zhang, M. Baker, and H. Sun, "Dynamic icic in lteadvanced networks for inter-cell interference mitigation," in 2015 IEEE 82nd Vehicular Technology Conference (VTC2015-Fall), Sep. 2015, pp. 1-5. doi: 10.1109/VTCFall.2015.7390903

[19] N. Trabelsi, L. Roullet, and A. Feki, "A generic framework for dynamic eicic optimization in lte heterogeneous networks," in 2014 IEEE 80th Vehicular Technology Conference (VTC2014-Fall), Sep. 2014, pp. 1-6. doi: 10.1109/VTCFall.2014.6965814

[20] Y. Liu, C. S. Chen, and C. W. Sung, "Distributed enhanced inter-cell interference coordination (eicic) in lte-advanced hetnets: A potential game approach," in 2015 IEEE 81st Vehicular Technology Conference (VTC Spring), May 2015, pp. 1-5. doi: 10.1109/VTCSpring.2015.7145784

[21] K. Yao, Q. Wu, Y. Xu, and J. Jing, "Distributed abs-slot access in dense heterogeneous networks: A potential game approach with generalized interference model," IEEE Access, vol. 5, pp. 94-104, 2017. doi: 10.1109/ACCESS.2016.2614701

[22] M. Boujelben, S. B. Rejeb, and S. Tabbane, "A comparative study of interference coordination schemes for wireless mobile advanced systems," in The 2014 International Symposium on Networks, Computers and Communications, June 2014, pp. 1-5. doi: 10.1109/SNCC.2014.6866529

[23] G. T. R. W. (2010), "R1-104355: Potential performance of range expansion in macro-pico deployment," p. 104, 2010.

[24] N. Trabelsi, C. S. Chen, R. E. Azouzi, L. Roullet, and E. Altman, "User association and resource allocation optimization in lte cellular networks," IEEE Transactions on Network and Service Management, vol. 14, no. 2, pp. 429-440, June 2017. doi: 10.1109/TNSM.2017.2677778

[25] M. S. Ali, "An overview on interference management in 3gpp lteadvanced heterogeneous networks," in International Journal of Future Generation Communication and Networking, vol. 8, no. 1, 2015, pp. 55-68. doi: $10.14257 /$ ijfgen.2015.8.1.07

[26] D. Monderer and L. S. Shapley, "Potential games," Games and Economic Behavior, vol. 14, no. 1, pp. 124 - 143, 1996. doi 10.1006/game.1996.0044

[27] A. B. MacKenzie and L. A. DaSilva, Game Theory for Wireless Engineers (Synthesis Lectures on Communications). Morgan \& Claypool Publishers, 2006.

[28] F. Capozzi, G. Piro, L. A. Grieco, G. Boggia, and P. Camarda, "Downlink packet scheduling in lte cellular networks: Key design issues and a survey," IEEE Communications Surveys Tutorials, vol. 15, no. 2, pp. 678-700, Second 2013. doi: 10.1109/SURV.2012.060912.00100

[29] S. Sesia, I. Toufik, and M. Baker, LTE, The UMTS Long Term Evolution: From Theory to Practice. Wiley Publishing, 2009.

[30] M. T. Kawser, H. M. A. B. Farid, A. R. Hasin, A. M. J. Sadik, and I. K. Razu, "Performance comparison between round robin and proportional fair scheduling methods for lte," in International Journal of Information and Electronics Engineering, vol. 2, no. 5, September 2012, pp. 678671. doi: 10.7763/IJIEE.2012.V2.186 
[31] J. Moreira, E. Silva, and P. Guardieiro, "Técnicas de coordenação de interferência inter-celular para redes heterogêneas no downlink lte-a," in Anais do XXXVII Simpósio Brasileiro de Redes de Computadores e Sistemas Distribuídos. Porto Alegre, RS, Brasil: SBC, 2019, pp. 890-903. doi: $10.5753 /$ sbrc.2019.7410

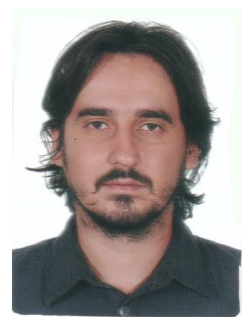

Júnio Moreira is Professor EBTT on Instituto Federal do Triângulo Mineiro of Patrocínio, Brazil, and researcher at the laboratory of computer networks from UFU, where he develops research on LTE-A HetNets/5G. He received his B.Sc. in Information Systems from at Pontifical Catholic University of Arcos and obtained his M.Sc. in Systems Engineering from University of Lavras. Currently, he is pursuing the Ph.D. degree with the Institute of Engineering at University of Uberlândia (FEELT/UFU). His current research interests include: computer networks, Heterogeneous Networks, game theory, inter-cell interference coordination techniques, quality of service (QoS), sheduling and simulations.

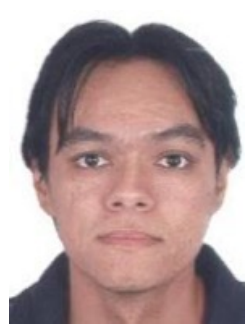

Éderson Rosa da Silva is an Associate Professor at Universidade Federal de Uberlândia (UFU), Brazil, where he has worked since 2011. He received his B.E. degree in Electrical Engineering from UFU in 2007 and the Ph.D. degree in Electrical Engineering from UFU in 2010. His research interests include performance analyses of computer networks, mobile communications and quality of service (QoS).

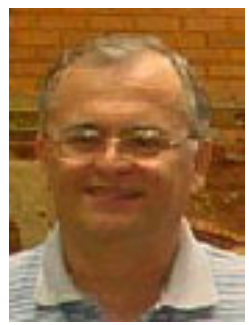

Paulo Roberto Guardieiro is a Full Professor at Federal University of Uberlândia, Brazil, where he works since 1978. He received his B.E. degree in Electrical Engineering from UFU (Universidade Federal de Uberlândia) in 1978, the degree of M.E.E. from the ITA (Instituto Tecnológico de Aeronáutica), in 1984 and the Ph.D. degree in Electrical Engineering from the UNICAMP in 1991. He is coordinator of the Computer Networks Laboratory of the UFU and member of the Brazilian Society of Telecommunications (SBrT). His current research interests include the design, development, and performance evaluation of communication protocols and systems for LTE-A and 5G networks. 